\title{
Kutane Langerhanszell-Histiozytose bei Erwachsenen
}

\section{Cutaneus Langerhans-Cell-Histiocytosis in Adults}

\section{Zusammenfassung}

Bei der Langerhanszell-Histiozytose (Synonyme: Histiozytose X, Abt-Letterer-Siwe-Syndrom, Morbus Hand-Schüller-Christian, eosinophiles Granulom) handelt es sich um eine Reihe von Erkrankungen, die durch eine neoplastische Proliferation von Langerhans-Zellen mit Organinfiltration charakterisiert sind. Die Ätiologie ist ungeklärt. Es handelt sich um eine Erkrankung klonalen Ursprungs ohne sichere Hinweise auf Malignität. Die jährliche Inzidenz liegt bei 0,4/100 000 Kindern unter 15 Jahren, bei Erwachsenen liegt das Durchschnittsalter für die Erstmanifestation bei 33 Jahren.

Die definitive Diagnose der LCH erfordert den Nachweis von CD1a-positiven Langerhanszellen und/oder Bierbeck-Granula in der Elektronenmikroskopie.

Die Leitsymptome sind vielfältig und abhängig von der jeweiligen Organmanifestation der Erkrankung (80\% Knochenbefall, $60 \%$ Haut, Lunge, Leber, Knochenmark, ZNS, endokrinologische Beeinträchtigungen). Wir berichten den seltenen Fall einer Patientin mir einem Manifestationsalter jenseits der 80.

\section{Abstract}

The term Langerhans-cell histiocytosis (formerly known as histiozytosis X, Abt-Letterer-Siwe-Syndrom, Morbus Hand-Schueller-Christian, eosinophilic granuloma) encompasses a spectrum of diseases characterized by the neoplastic proliferation and infiltration of organs by langerhans-cells. The aetiology and pathogenesis of LCH remains largely undefined. It is considered to represent a clonal disease without definitive signs of malignancy. The incidence for children up to the age of 15 is $0,4 / 100000$, the mean age of first manifestation in adults is 33 years. Definitive diagnosis requires the demonstration of $\mathrm{CD} 1 \mathrm{a}$ antigenic determinants on the surface of lesional cells and/or the finding of Bierbeck granules in lesional cells by electron microscopy. The leading symptoms are determined by the different organ manifestations ( $80 \%$ bone, $60 \%$ skin, lung, liver, bone marrow, cerebral system, endocrinological symptoms). We report on the rare manifestation of this disease in a female patient beyond her eighties.

ist die LCH eine vor allem Kinder betreffende Erkrankung, die Datenlage zu Erwachsenen ist begrenzt. Leitlinien zur Diagnostik und Therapie bei Kindern sind u. a. über die Internetseite der $\mathrm{Ar}-$ beitsgemeinschaft der wissenschaftlichen medizinischen Fachgesellschaften (www.leitlinien.net) zugänglich [1]. 

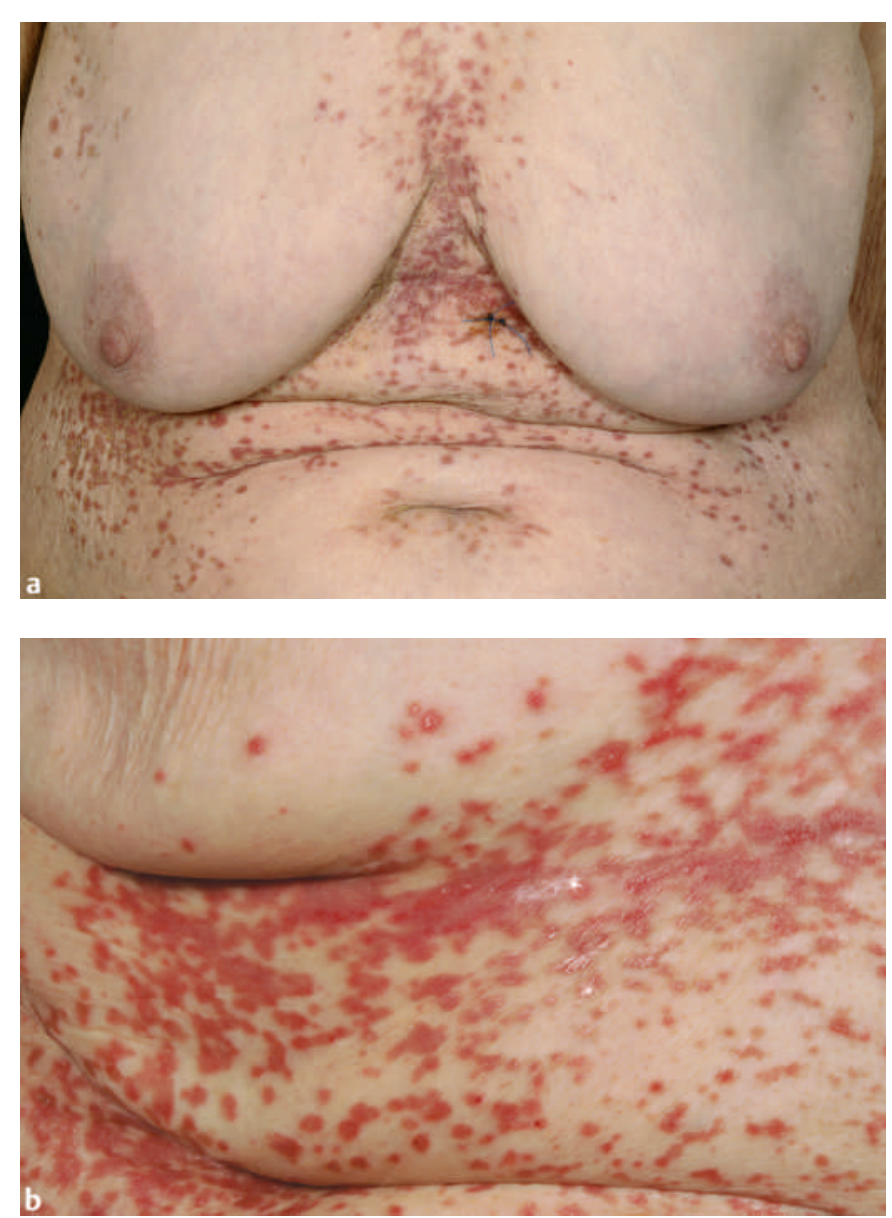

Abb. 1 a und b Kutane Langerhanszell-Histiozytose bei einer 82-jährigen Patientin: braunrote kleine monomorphe Knötchen im Stammbereich mit deutlicher Betonung der seborrhoischen und intertriginösen Areale.

\section{Kasuistik}

\section{Anamnese}

Bei der 82-jährigen Patientin waren seit ca. 3 Monaten Hautveränderungen in Form von roten, sukkulenten kleinen Papeln aufgefallen. Diese betrafen die Schulter- und LWS-Region sowie in ausgeprägterer und mehr konfluierender Form den inter- und submammären Bereich einschließlich des Bauchnabels.

Eine mit den Hautveränderungen einhergehende weitere Symptomatik wie Pruritus oder Schmerzen wurde nicht angegeben.

Als Nebendiagnosen sind kompensierte Herzinsuffizienz, KHK, stabile Angina pectoris, Mitralklappeninsuffizienz sowie anamnestisch eine TVT des linken Beines mit postthrombotischem Syndrom zu nennen.

Der Allgemeinzustand der Patientin war durch ihre kardialen Grunderkrankungen bestimmt und hatte sich in letzter Zeit nicht verschlechtert. Eine B-Symptomatik mit Gewichtsabnahme, Fieber und Nachtschweiß lag nicht vor.

Die Zuweisung erfolgte durch Herrn Dr. med. A. Müller-Schoch aus Schwäbisch-Gmünd mit ambulant durch Stanzbiopsie gesichertem Befund.
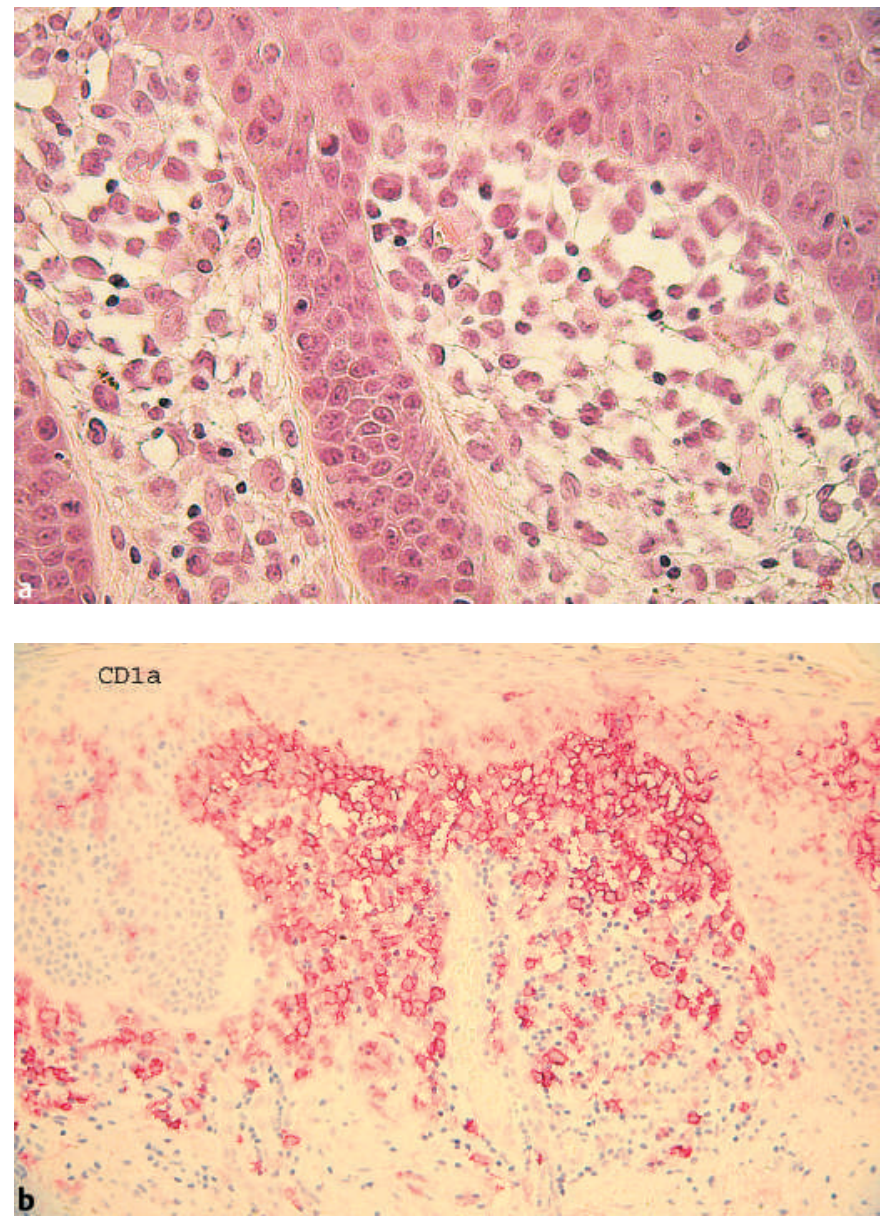

Abb. 2 Histologischer und immunhistologischer Befund. a Kompakte Infiltration des Stratum papillare mit hellen histiozytären Zellen mit parazentralen nierenförmigen Kernen. HE $\times 150$. b Deutliche Expression des Markers CD1a auf den Tumorzellen. Fast Red $\times 100$.

\section{Dermatologischer und körperlicher Aufnahmebefund}

Am Rumpf v.a. inter- und submammär sowie im LWS-, Schulterund Halsbereich teils dichte flächig konfluierende erythematöse Papeln, welche teilweise erhaben waren und teilweise im Hautniveau lagen (Abb. 1, 2).

Restliches Integument unauffällig.

\section{Labor}

Pathologisch verändert: GGT 60 U/l, Leukozyten $7400 / \mu l$ mit Monozytose (24,0\% Monozyten).

Normwerte für restliches Blutbild, Leber- und Nierenwerte, Elektrolyte.

\section{Apparative Untersuchungen}

Zum Ausschluss systemischer Beteiligung führten wir folgende Untersuchungen durch: Knochenmarkaspiration und Biopsie, HR-CT-Abdomen + Thorax, Extremitäten-Röntgen, ZMK-Konsil mit Zahnröntgen (Panorama), Schädel-MRT mit i.v. Kontrastmittelanreicherung, HNO-Konsilium mit Audiogramm, Gynäkologie-Konsil. 
In sämtlichen Untersuchungen konnten keine spezifischen pathologischen Befunde festgestellt werden.

\section{Histologische Befunde}

Zwei Biopsiestellen: Fokal parakeratotische Hornschicht mit Einlagerungen von Serum und Entzündungszellen. Im Stratum papillare dichte Konglomerate von histiozytären Zellen mit „reniformen“ parazentralen größeren Zellkernen und hellem Zytoplasma, welche fokal vermehrt auch die Epidermis durchsetzen. Die Zellen zeigen eine deutliche Positivität für CD1a.

Diagnose: Langerhanszell-Histiozytose.

\section{Therapie und Verlauf}

Die Patientin selbst lehnte eine medikamentöse Therapie ab. Da bei ihr ein rein kutaner Befall vorlag, führten wir eine Kurzzeittherapie mit Dermoxin-Creme durch und empfahlen für ambulant eine Lokaltherapie mit Kortikosteroiden und regelmäßige klinische Nachkontrollen.

\section{Diskussion}

Die LCH ist eine monoklonale Erkrankung, bei der pathophysiologisch ein interzellulärer Kommunikationsdefekt zwischen T-Zellen und Langerhanszellen angenommen wird. Trotz des klonalen Ursprungs ist die Malignität der Erkrankung nicht eindeutig belegt, zumal es in der Literatur Hinweise auf Spontanremissionen gibt [1-4].

Die Schwere und Symptomatik sowie auch die Prognose der Krankheit wird bestimmt durch die Ausdehnung und Lokalisation des Organbefalls. So kann z. B. der Befall der Hypophysenregion zur Ausbildung von endokrinologischen Defiziten i.S. eines Diabetes insipidus führen.

Die in Tab. 1 genannten Therapieoptionen stehen zur Verfügung $[1,2]$.

In einer Fallstudie von F. Giona et al. wurden 11 Patienten mit unterschiedlichem Befall mit den oben beschriebenen Therapieoptionen behandelt. Dabei wurden mit den Lokaltherapien zwar vorübergehend gute Erfolge erzielt, es kam jedoch häufig zu Rezidiven bzw. Manifestationen an anderen Lokalisationen. Hierbei ist sicherlich auch in Frage zu stellen, inwieweit das vorherige
Tab. 1 Therapieoptionen bei Langerhanszell-Histiozytose

\begin{tabular}{|c|c|}
\hline Lokaltherapie & $\begin{array}{l}\text { topische Kortikosteroide, } 20 \% \text { Nitrogen-Mustard- } \\
\text { Lsg, intraläsionale Kortikoid-Instillation lokalisierter } \\
\text { Haut- oder Knochenbefall }\end{array}$ \\
\hline chirurgische Therapie & v. a. bei lokalisiertem Befall in Knochen und Lunge \\
\hline Strahlentherapie & $\begin{array}{l}\text { Kumulativdosis max. 6-10 Gy } \\
\text { nur bei speziellen Indikationen wie z. B. schnell } \\
\text { wachsende Raumforderung im Hypophysenbereich }\end{array}$ \\
\hline Chemotherapie & $\begin{array}{l}\text { Vinblastin, Etoposid (+ Methylprednisolon) } \\
\text { systemischer Befall mehrerer Organe }\end{array}$ \\
\hline Immuntherapie & $\begin{array}{l}\text { Interferon } \\
\text { lokalisierter Befall (intraläsionale Injektion) oder } \\
\text { systemischer Befall (s.c. Applikation) }\end{array}$ \\
\hline Thalidomid & $\begin{array}{l}\text { antiangionetisch, antineoplastisch, } \\
\text { immunmodulatorisch } \\
\text { systemischer Befall mehrerer Organe }\end{array}$ \\
\hline
\end{tabular}

Staging effizient genug war. Die systemischen Therapien dagegen hatten meist einen langfristigen Effekt, der sicherlich auch damit zu begründen ist, das etwaige vorher nicht erkannte Herde mitbehandelt worden sind [2].

Da wir bei der Patientin eine systemische Beteiligung ausschließen konnten und sie zudem eine systemische Therapie ablehnte, entschlossen wir uns zu einer Lokaltherapie mit externen Kortikosteroiden, beginnend mit einem Klasse-IV-Steroid und anschließender Reduktion auf Klasse II. Alternativ wäre auch ein zuwartendes Verhalten ohne Therapie zu diskutieren vor dem Hintergrund einer möglichen Spontanremission. Aktuell ist der Hautbefund konstant.

\section{Literatur}

${ }^{1}$ Leitlinie Langerhanszell-Histiozytose der Arbeitsgemeinschaft wissenschaftlichen medizinischen Fachgesellschaften. Stand Juni 2000 (www.leitlinie.net)

${ }^{2}$ Giona $\mathrm{F}$ et al. Langerhans cell histiozytosis in adults. A clinical and therapeutic analysis of 11 patients from a single institution. Cancer 1997; 80: 1786- 1791

${ }^{3}$ Arico $\mathrm{M}$ et al. Langerhans cell histiocytosis in adults. Report from the International Registry of the Histiocyte Society. Europ J Cancer 2003; 39: $2341-2348$

${ }^{4}$ Donadieu J. Langerhans cell histiocytosis: portrait of the disease as a rare tumor. Europ J Cancer 2004; 40: 1465 - 1466 